\title{
TOWARDS FUNCTIONAL ARCHITECTURAL DECOMPOSITION OF CONSTRAINED LAYER INFLATABLE SYSTEMS
}

\author{
Benli, Koray (1); \\ Luntz, Jonathan (2); \\ Brei, Diann $(1,2)$; \\ Kim, Wonhee (3); \\ Alexander, Paul (3); \\ Johnson, Nancy (3) \\ 1: Design Science, University of Michigan; \\ 2: Mechanical Engineering, University of Michigan; \\ 3: General Motors R\&D
}

\begin{abstract}
Pneumatically activated systems enable myriad types of highly functional inflatables employing a wide range of architectural approaches affecting their form and function, making systematic conceptual design difficult. A new architectural class of pneumatically activated systems, constrained layer inflatable systems, consists of hierarchically architected flat layers of thin airtight bladders that are internally and/or externally constrained to generate a variety of functionalities. The highly hierarchical architectural structure of constrained layer inflatable systems coincides with the hierarchy of produced functions, providing an opportunity for the development of a functional architectural decomposition, capturing the inherent relationship between architectural and functional hierarchies. The basis of the approach is conveyed through the design of an example constrained layer inflatable system. This approach empowers the systematic understanding of the interrelated architectural and functional breakdown of constrained layer inflatable systems, enabling designers to iteratively analyze, synthesize, and re-synthesize the components of the system improving existing designs and exploring new concepts.
\end{abstract}

Keywords: Hierarchical decomposition methods, Form and function, Design methods, Product architecture, Technology

\section{Contact:}

Benli, Koray

UNIVERSITY OF MICHIGAN

Design Science

United States of America

bkoray@umich.edu 


\section{INTRODUCTION}

Pneumatically activated inflatable devices have been proven useful in various fields due to the technology capabilities they provide such as distributed actuation, changes in structural properties, and distributed sensing. In the medical field, capabilities such as moldability, repositioning, variable stiffness, and actuation have been used across applications including medical casts (Mitsuda and Matsuo, 2005), wheelchairs (Veneman et al., 2015), rehabilitation, (Sadeghi et al., 2019), and muscle actuators. The aerospace and civil engineering fields have developed lightweight deployable structures such as antennas (Freeland et al., 1997) and space structures (Seedhouse, 2015), as well as temporary flood control dams (Gurt, 2015). In the automotive field, inflatable systems capable of morphing and texturization have been explored for external aerodynamic surfaces (Terwagne et al., 2014), and driver information and alert systems (Tome, 2015). Finally, the human computer interaction field has demonstrated pneumatically activated devices for interactive displays with flexible (Matoba et al., 2012), malleable (Follmer et al., 2012), and reconfigurable, controllable stiffness controls on pressuresensitive touch surfaces (Han et al., 2020).

These pneumatically activated systems use a wide variety of architectural approaches which impact their form and function. Typically, the design of such systems is done in an ad hoc manner because they are generally free-form in structure. A new architectural class of pneumatically activated systems is constrained layer inflatable systems which are formed from architectures consisting of many flat layers of thin airtight bladders arranged hierarchically, and individually or mutually constrained both internally and externally. The huge variety of these architectural constraints, both internal (tiles, tendons, etc.) and external (plates, interconnections, etc.) produces the high degree of functionality enabled by constrained layer inflatable systems such as different modes of actuation, changes in structural properties, and sensing capabilities. This architectural variety is structured in a hierarchical manner, where basic functions produced by basic architectural elements combine in ensembles and subassembly units to produce higher level aggregate and compound functions, and ultimately technology capabilities of a system which enable an application. This structure creates the opportunity to enable systematic design by relating the structured arrangement of the architectural components embedded in a constrained inflatable system to the corresponding structured set of functions. The debate on defining the relation between form and function is an age-old question that can be retraced to Sullivan's (1896) dictum "form ever follows function". Instead of spending effort towards unravelling the causality between form and function, the highly structured nature of constrained layer inflatable systems allows for the coexistence of form and function, which are almost perfectly overlapping in a hierarchical manner.

This paper proposes an integrated hierarchical characterization scheme: functional architectural decomposition, geared towards supporting various analysis and synthesis design methods. It relies on the coherence between the architectural and functional hierarchy inherent in constrained layer inflatable systems. By mapping architectural components to the corresponding functions, compound functions can be directly produced from the aggregation of compound architectural components, where basic functions of cells combine to form aggregate functions in ensembles of cells. Ensembles combine to produce more complex compound functions within a unit, and units combine in assemblies to form a complete system providing technology capabilities to enable applications. The general architecture detailing the fundamental components of a basic constrained layer inflatable system is presented. The architecture of three particular constrained layer inflatable example systems demonstrate a wide range of capabilities provided by constrained layer inflatable systems. The characterization scheme and the implementation of the functional architectural decomposition is communicated through a mobile phone restraint mat design example. This scheme provides a direct view into the hierarchical structure of a system's form and function geared toward enabling analysis, synthesis, and re-synthesis at a conceptual level.

\section{CONSTRAINED LAYER INFLATABLE SYSTEMS}

The constrained layer inflatable system architecture enables the design of highly customizable, lightweight, space saving, and low-cost inflatable systems. They are composed of multiple thin flat airtight bladder layers which encapsulate inflatable air cavities, the inflation of which are constrained both internally and/or externally to produce various functions. These are arranged in layered hierarchical architectures that correspond to a hierarchy of functions. When these systems are exposed to a relative 
pressure change, they exhibit various technology capabilities such as stiffness changing, deploying/stowing, texturization, actuation, etc. Three examples demonstrate the fundamental architectural components and the ways of combining them to form constrained layer inflatable systems.

\subsection{Constrained Layer Inflatable Architecture}

The basic architecture of a constrained layer inflatable system (Figure 1) is composed of one or multiple cells made of 1) airtight cell skin layers that encapsulate an inflatable air cavity, 2) a pneumatic port, and 3) internal or external constraints. The top and bottom skin layers of a cell are stacked and permanently sealed to enclose the air cavity. A vacuum port attached to one of these cell skin layers allows control over pressure inside the layered structure. Based on the pressure level variations in a cell and the architectural design of a cell, the cell produces basic functions such as different modes of actuations (out of plane expansion, in plane contraction), changes in structural properties (rigidity and extensibility), and sensing capabilities (contact pressure, proprioception, or environmental conditions). The architectural design of a cell, particularly the constraints, both internal (rigid tiles, tethers, etc.) and external (inter-cell and external structural connections, for example), create the basic functions it produces through its operation as the pressure level changes.

An individual cell can be mechanically and pneumatically connected to a multiplicity of identical, or modified cells in a stacked (vertical) or in-plane (horizontal) fashion, generating various forms of cell ensembles. Because an ensemble is typically formed from cells with similar basic functions, an ensemble often combines the basic functions of the cells that constitute them to create aggregate functions which are generally amplified or extended versions of the basic functions. For example, an ensemble comprising a vertical stack of cells, each of which can expand with a small degree of angular motion, aggregates the individual angular motions into a combined large scale aggregate angular expansion, combining the effects of the individual cells. Thus, the hierarchical aggregation of the architectural form of a constrained layer inflatable system coincides with the aggregation of its functional capabilities.

Differently generated cell ensembles can be assembled together in myriad ways to produce units. Since the aggregate functions of the ensembles that make up a unit can be vastly different, new emergent compound functions can be produced. For example, an ensemble producing aggregate angular expansion assembled with unit producing aggregate linear expansion can deploy from a flat surface and push an object. These units can be combined to form an assembly of units, which further creates higher level compound functions (for example, two opposing pushes in an assembly create a squeeze). In general, assemblies can themselves act as sub-assemblies in a higher-level assembly and eventually constitute the design of a particular, potentially multifunctional, constrained layer inflatable system that produces one or more technology capabilities to enable an application.

To illustrate the variety of cells, ensembles, units, assemblies, and systems and the corresponding basic functions, aggregate functions, compound functions, and technology capabilities that constrained layer inflatables can produce, three examples are given: 1) a deployable mobile phone restraint mat which employs a hierarchy of two assemblies of two units, each unit comprising two ensembles of cells to position and orient a phone on a wireless charger, 2) internally tiled active textiles which are made of a single 2D horizontal ensemble of tiled cells to shape and rigidize over an object to constrain it, and 3) a variable friction cargo restraint surface made up of two interdigitated sets of 1D horizontal ensembles to change friction properties to selective allow (for loading) or restrict (for transit) sliding of objects.

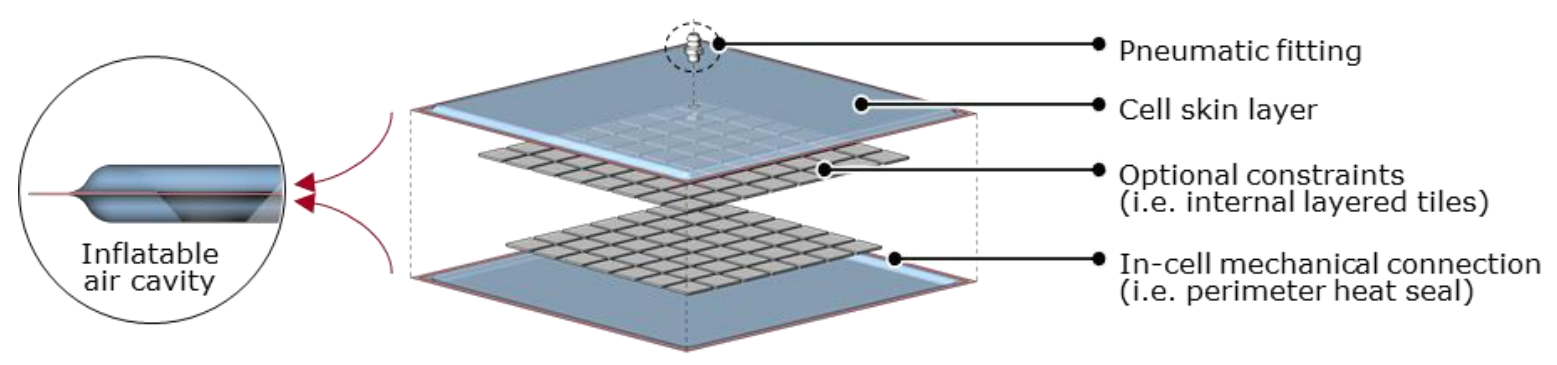

Figure 1. Example constrained layer inflatable system architecture consisting of top and bottom cell skin layers, an inflatable air cavity, tile constraints, and a pneumatic fitting 


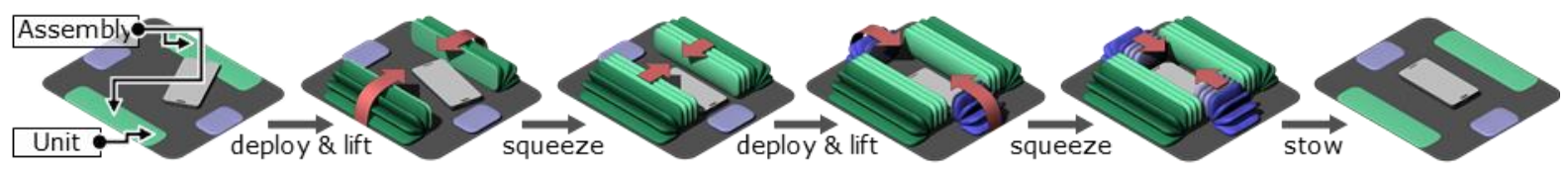

Figure 2. Assemblies of the mobile phone restraint mat sequentially orienting a cell phone in an appropriate position on the charging pad by activating a sequence of the provided technology capabilities: deploying, lifting, squeezing, and stowing

\subsection{Example Architecture 1: The Mobile Phone Restraint Mat}

The mobile phone restraint mat automatically positions and orients a cell phone over an inductive charger using an embedded constrained layer inflatable system providing the technology capabilities of deploying, lifting, squeezing, and stowing. The system's architecture consists of two separate orthogonally oriented deployable and stowable assemblies attached to a charging mat. The assemblies have the capabilities of lifting and squeezing the mobile phone and thereby can move and align it on the pad using a sequence of these capabilities (Figure 2).

Each one of the orthogonal assemblies (Figure 3b) is made of opposing, pneumatically interconnected units, and each of these is made of two vertical cell ensembles that are stacked on top of each other (Figure 3c). These units deploy and stow in sequence and provide the compound functions of angular deployment and linear pushing (where two units pushing together creates the capability of a squeeze). Two vertical ensembles form each of these units, the first of which (Figure 3d) attaches to the base mat and produces aggregate angular expansion and contraction while the second attaches to the first and provides aggregate linear expansion and contraction. Each cell within one of these ensembles (the first, for example, Figure 3e), is customized to form the vertical cell ensemble by providing specific basic functions such as attachment to the base and to the next cell in addition to angular expansion.

The sub-cellular architecture of one particular cell (Figure 3f) consists of top and bottom cell skin layers encapsulating an inflatable air cavity in between, and a pneumatic port attached to the bottom cell skin layer through a hole through the external surface constraint to which the bottom cell skin layer is permanently bonded. The bottom cell skin layer of the next adjacent cell is mechanically connected to the top cell skin layer of the previous cell in the ensemble around the opening highlighted with green (Figure 3f). The resulting mobile phone restraint mat provides an effortless way to position any mobile phone over a wireless charger while stowing flush to the surface when not in use.

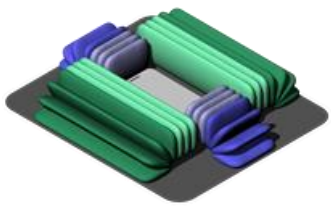

a) System I. \& II.

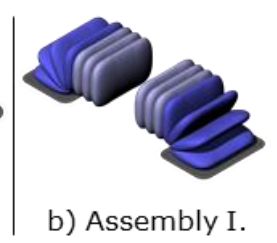

b) Assembly I.

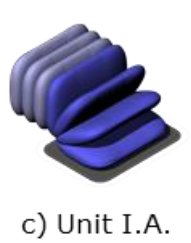

c) Unit I.A.

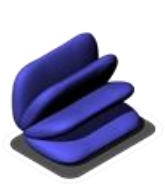

d) Ensemble I.A.1.

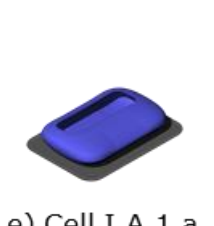

e) Cell I.A.1.a.

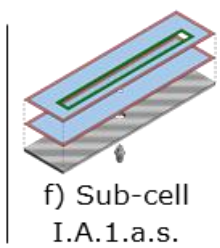

I.A.1.a.s.

Figure 3. An exemplary partial architectural decomposition of the mobile phone restraint mat

\subsection{Example Architecture 2: Internally Tiled Active Textiles}

A second example is internally tiled active textiles that provide thin-profile surfaces that can be draped over arbitrary objects, shaped into complex geometries, and rigidized to keep the target form (Figure

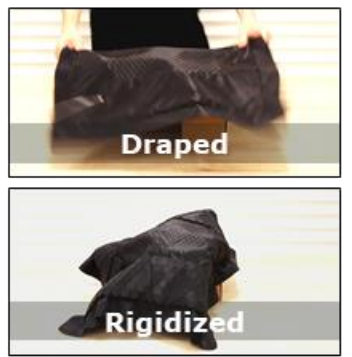

a)Technology capabilities

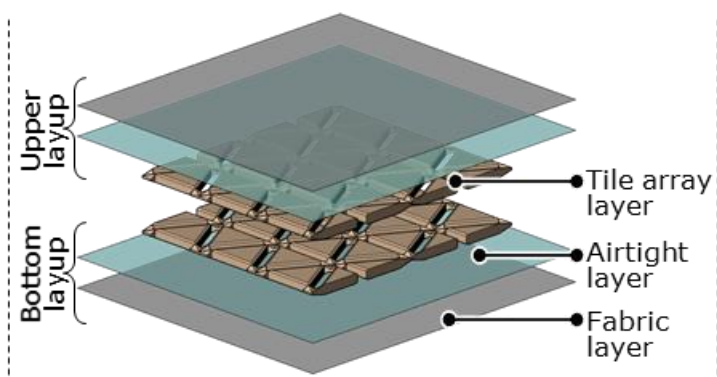

b) Constrained layer inflatable device

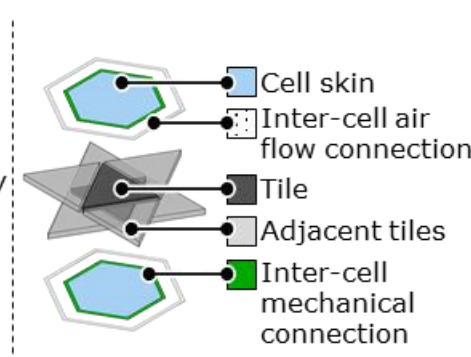

c) Example cell

Figure 4. Active textile prototype demonstrating drapability and rigidizability technology capabilities (a), an example generic architecture of the internally tiled active textiles (b), and an example cell (c) 
4a). The architecture is made up of horizontally assembled variations of cells equipped with internal rigid tile constraints. Each cell comprises a pair of flat opposing tiles sandwiched between airtight bladder layups (Figure 4c). Normally, the opposing tiles allow relative motion: sliding and rotating out of plane. When a vacuum is applied between the bladder layers, the tiles jam against each other, constraining their relative position. When arrayed in a two-dimensional ensemble over the entire surface (which also forms a single unit making up the entire system - Figure $4 b$ ), the relative motion aggregates into a draping capability, and the freezing in place aggregates into the shaping and rigidizing capabilities. This system enables applications such as a cargo retention blanket or an emergency medical cast.

\subsection{Example Architecture 3: Variable Friction Surface}

A third example of a constrained layer inflatable system is a variable friction surface capable of changing its surface properties between high and low friction states (Figure 5a) and is made up of two interdigitated units (Figure 5b). The low friction unit is a horizontal combination of a number of horizontal linear ensembles (rows) of circular cells (Figure 5c), each of which has the basic function of out-of-plane expansion. Together, the cells in the low friction unit (each made of a low friction material) have the capability to support the weight of an object and provide a low friction surface when inflated. Similarly, the angular shaped cells making up the horizontal ensembles of the high friction unit have a rubberized coating and have the capability of lifting the object and providing a high friction surface when inflated. These two units form an interdigitated assembly, which makes up the entire system providing the variable friction capability. The variable friction surface can be used on the floor of a storage compartment of a vehicle, allowing easy loading of items in the low friction mode, while helping to hold them in place during transport in the high friction mode.

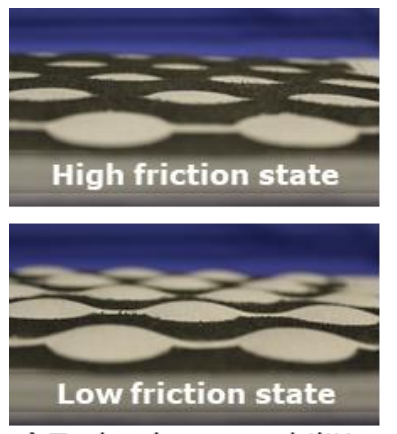

a) Technology capabilities

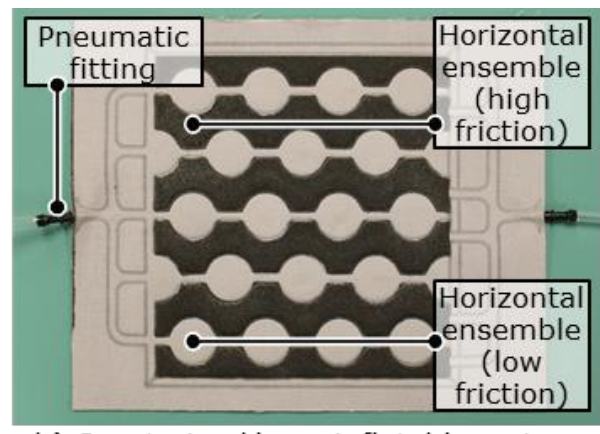

b) Constrained layer inflatable system

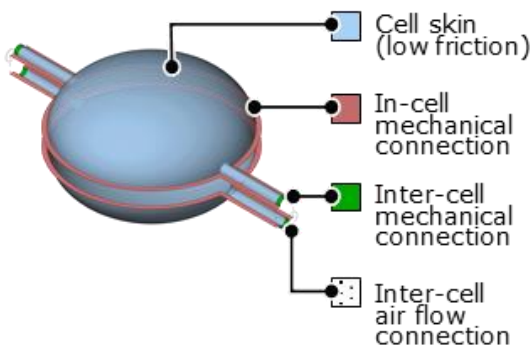

c) Example cell

Figure 5. The variable friction cargo surface system for vehicles demonstrating low-high friction modes as its technology capabilities

\section{FUNCTIONAL ARCHITECTURAL DECOMPOSITION APPROACH}

The goal of the functional architectural decomposition is to provide a basis for the iterative analysis and synthesis of the relation between the hierarchical architectural form of a design and the corresponding hierarchical functional capabilities. It allows the decomposition of a constrained layer inflatable system to the lowest level where basic functions are provided by the smallest architected set of components. It also enables synthesis at higher levels, where the basic functions (i.e. angular expansion) can be aggregated to produce aggregate functions (i.e. aggregate angular expansion), which form compound functions (i.e. angular deployment) when combined at higher levels, eventually producing technology capabilities (i.e. lifting and squeezing) at the highest level of the functional architectural decomposition. This hierarchy matches the hierarchy of the (sub-cellular) components that make up the basic architectures (cell) for producing aggregated (ensemble) and compound (unit) architectures, where each architectural component at any level correspond one or more basic, aggregate, or compound functions. As an illustrative example, the architectural decomposition of the mobile phone restraint mat is shown in Figure 6 which provides context for the description of the approach throughout the levels. Since the structure is fundamentally hierarchical, the various architectural components are labelled in outline form according to this hierarchy (I., I.A., I.A.1. etc.). Due to their highly structured architectural formation, constrained layer inflatable systems provide an ideal context for the development of the functional architectural decomposition which aims to aid 


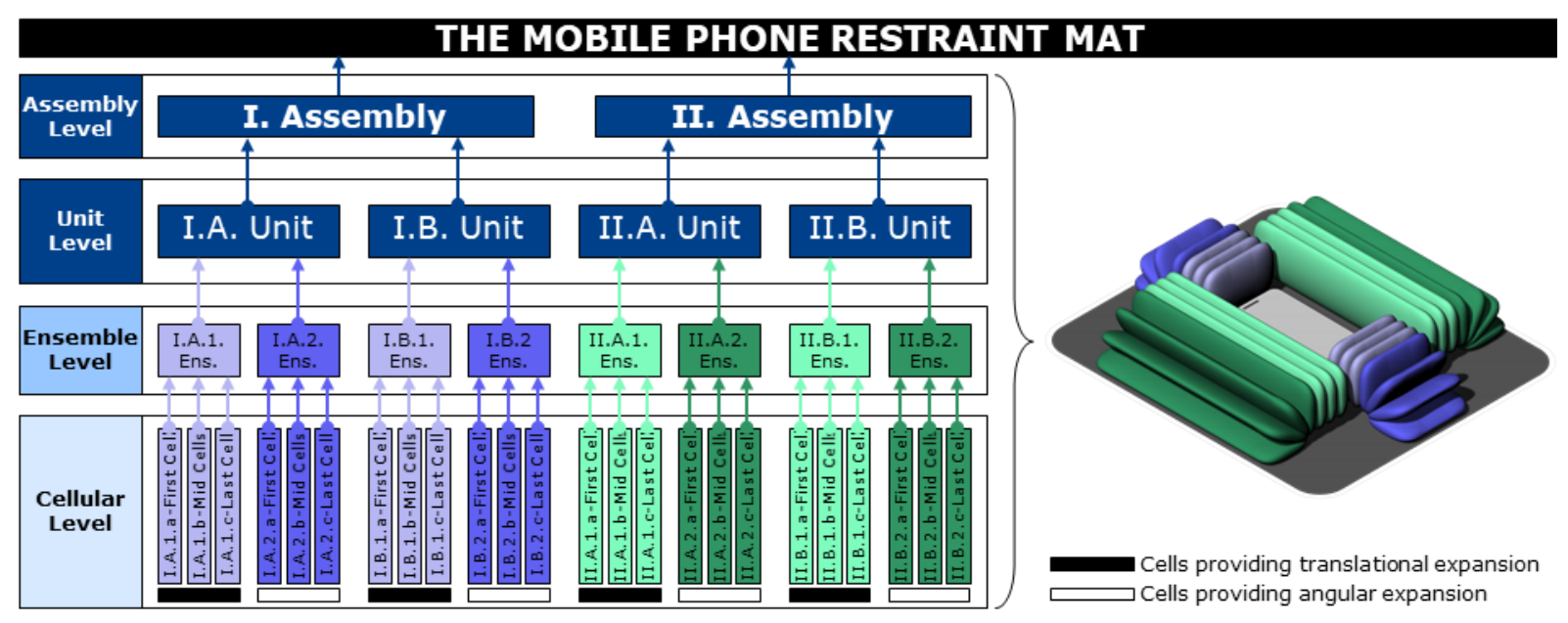

Figure 6. Architectural decomposition of the mobile phone restraint mat as a motivational example. Inherent hierarchical structure is also indicated by outline form labelling.

exploration of design alternatives by analysis, synthesis, and re-synthesis of the decomposition. There are four main levels forming the functional architectural decomposition: 1) sub-cellular level, describing the properties of the components of a cell, 2) cell level, describing the architectural details of a cell and the corresponding basic functions, 3) ensemble level, describing the ways a set of cells is assembled to form a cell ensemble providing aggregate functions, 4) unit level, describing how cell ensembles are brought together to form a unit providing compound functions and 5) assembly level(s), associated closely with the unit level, describing how multiple units (or sub-assemblies) come together to produce a constrained layer inflatable system with certain technology capabilities. A bottom-up approach used in the context of the mobile phone restraint mat example to describe the architectural and functional hierarchy through the levels along with a brief discussion of how the functional architectural decomposition supports design analysis, synthesis, and resynthesis.

\subsection{Sub-cellular Level}

The sub-cellular level enumerates three feature categories: characteristics of cell skin layers, rules of connectivity affording cell and ensemble construction, and details regarding constraints. The properties of these features together determine the function(s) that a cell provides.

There are three major subcategories forming the characteristics of a cell skin layer: skin type, skin thickness, and skin uniformity. Skin type classifies the potential materials that can be used as cell skin layers based on two levels of physical extensibility (extensible - inextensible) and three levels of physical flexibility (low-medium-high). For example, a bare sheet of thermoplastic polyurethane (TPU) would be classified as an extensible material with high flexibility, whereas a relatively thick polypropylene sheet would be classified as inextensible material with low flexibility. Skin thickness applies to the top and bottom cell skin layers, indicating whether a cell is architecturally symmetric or not. Skin uniformity specifies whether the constituents of a cell layup are uniform or non-uniform (i.e. changing cross-section thickness) transversally.

The rules of connectivity that affords cell and ensemble construction are grouped under four categories: in-cell mechanical connection, inter-cell mechanical connection, inter-cell pneumatic connection, and extrinsic connectivity. The in-cell mechanical connection describes the permanent bonding between top and bottom cell skin layers, forming an enclosed cell or a cell with a designated opening or openings around its perimeter. The inter-cell mechanical connection describes how adjacent cells can be permanently bonded together in order to form a cell ensemble. Inter-cell pneumatic connection describes the back-and-forth air flow direction between cells as the pressure inside the layered structure changes in relation to atmospheric pressure. The extrinsic connectivity details the way and the location of a pneumatic fitting attached to one of the cell skin layers to enable air flowing in and out of the corresponding cell.

There are two main categories of constraints, internal and external. Internal constraints include volumetric, layered, and tendon-like constraints. Using particles such as glass beads or coffee beans encapsulated in a cell to produce a controllable stiffness change as a function of vacuum pressure, or accommodating materials such as sponge or strands of twisted fibers to control the air flow velocity can 


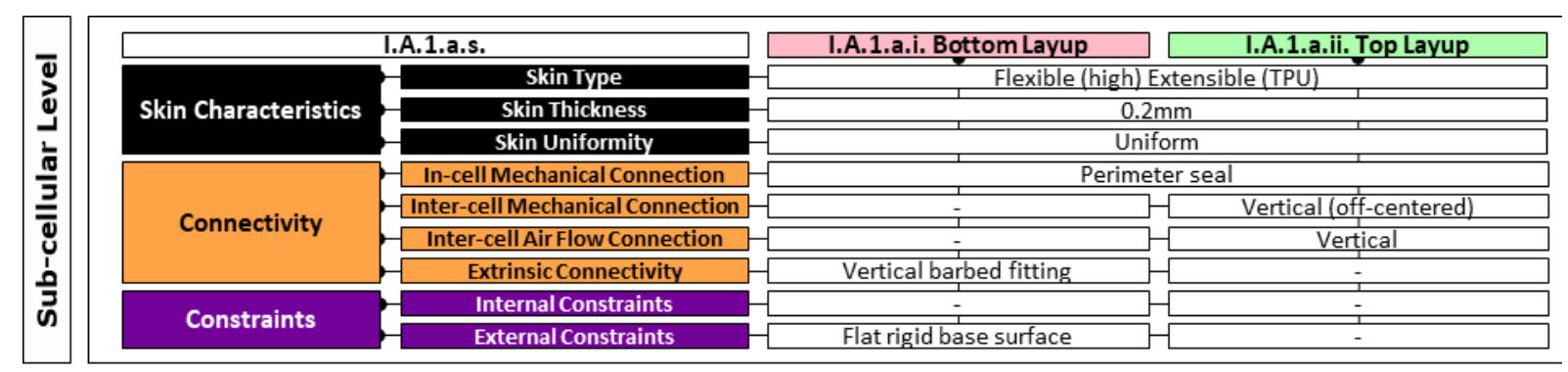

Figure 7. Sub-cellular features of one of the cell variations (I.A.1.a.s.) that constructs the mobile phone restraint mat

are examples of internal volumetric constraints. Replacing the bulky particles filling a cell with thin uniform sheets such as paper or textile that are layered on top of each other, or using one or multiple layers of non-uniform yet regularly arrayed basic or architected tile patterns are examples of internal layered constraints. Accommodating strings or cables attached between cell skin layers to restrain the inflation of a cell is an example of internal tendon-like constraints, which can be in a discrete or distributed form. External constraints can be surface, boundary, or tendon-like constraints. In the case of using a constrained layer inflatable system as an actuator, at least one of the cells needs to be attached to a surface that acts as an external surface constraint. External boundary constraints can be used to restrain a cell around its perimeter or a portion of its perimeter to facilitate a designated physical behavior such as limiting the out of plane translational expansion or allowing out-of-plane curling around a fixed axis. External tendon-like constraints are the equivalent internal tendons, but situated outside a cell with strings or cables attached to the exterior surface of at least one of the cell skin layers.

The sub-cellular features of one of the particular cells (I.A.1.a.) that make up the mobile phone restraint mat are compactly represented in categorical tabular form as an example in Figure 7. Skin characteristics are shared between the top and bottom layups that constitute this particular cell, whereas the constraints and some of the connectivity features vary between top to bottom layup. These specified features provide a basis for cell and ensemble formation at the higher levels of the functional architectural decomposition.

\subsection{Cell Level}

The cell level catalogues the types of cells, cell variations, and the basic functions they provide, enabling the embodiment of a constrained layer inflatable system. Any cell can be architecturally modified in many ways, generating a cell variation, which either replicates or modifies the existing basic function or introduces an alternative one. The structures that enable the organization among different types of adjacent cells or cell variations are detailed at the cellular level. The nature or the number of basic functions emerging from the sub-cellular construction shows similarities or differences depending on the architectural compositions and sub-cellular features of cells.

In the mobile phone restraint mat example, there are three cell variations within the particular cell ensemble (I.A.1.): first (I.A.1.a.), mid (I.A.1.b.), and last cells (I.A.1.c.) (Figure 8). Each variation has slightly different sub-cellular features, providing slightly different sets of basic functions. For example, only the first cell can "connect to external air source" and has a constraint to "attach to flat surface", whereas only the last cell can "attach (mechanically) to next cell ensemble". The basic functions enabling mechanical and pneumatic attachment to the next and/or previous cells, as well as providing angular contraction and expansion are shared among all cell variations.

The inception of each one of these basic functions provided by cell variations can be traced back via provisional arrows (color-coded to the sub-cellular feature categories) to the features of each cell at their sub-cellular level (Figure 9). By changing the sub-cellular features, the basic functions of a cell can be adjusted or substituted with new functions.

\subsection{Ensemble Level}

The ensemble level describes the collection of cells that are assembled together to provide aggregate architectural components, and corresponding aggregate functions. Assembling multiple cells in a stacked fashion generates a vertical ensemble, and assembling multiple cells side by side on a plane generates a horizontal ensemble. The ability to assemble multiple cells in different ways to generate vertical or horizontal ensembles resides in the sub-cellular features of those cells. By making necessary changes 

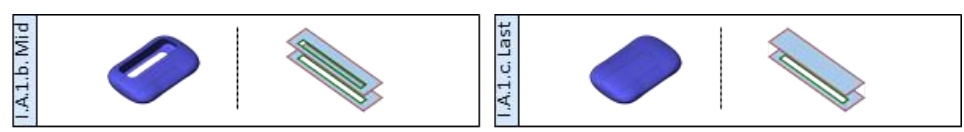

Figure 8. A set of cell architectural variations (I.A.1.a., I.A.1.b., and I.A.1.c.) that form a particular ensemble (I.A.1.) of the mobile phone restraint mat system
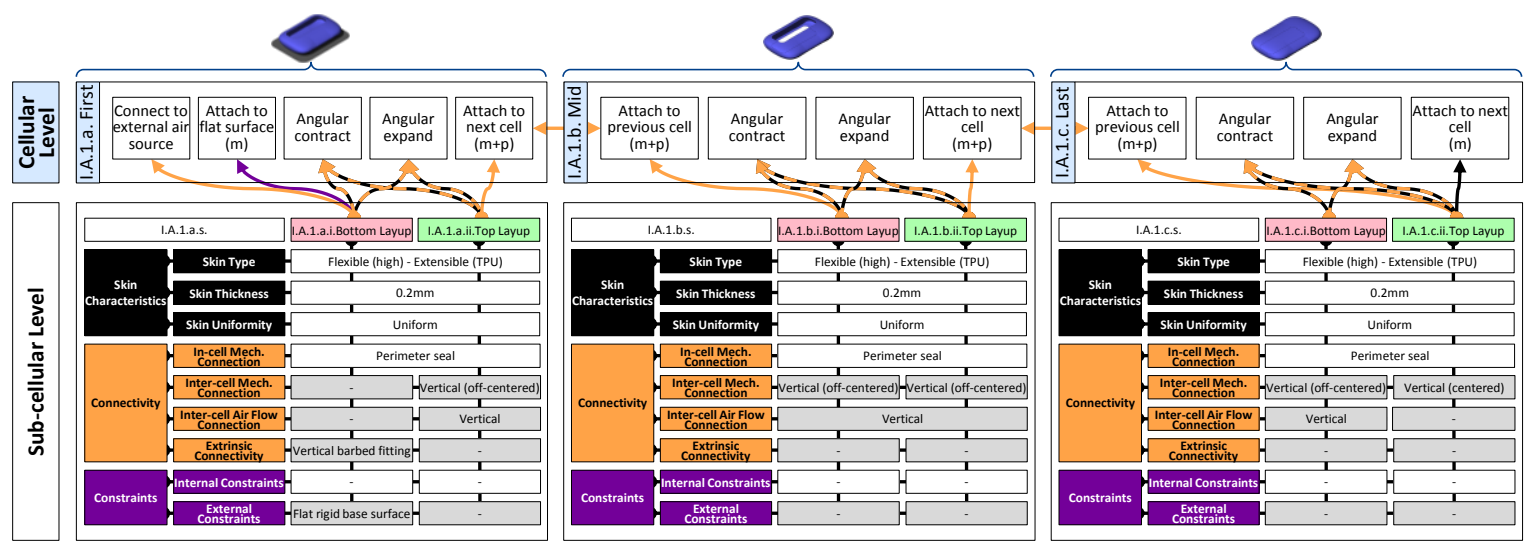

Figure 9. Sets of basic functions traced back to the sub-cellular features provided by each cell variation at the cellular level of decomposition

regarding the inter-cell mechanical and inter-cell pneumatic connectivity characteristics of cells in their sub-cellular levels, it is possible to pave the way for the construction of any of these two types of cell ensembles. The architectural differences between the two types of cell ensembles does not necessitate a functional diversity. The same aggregate function can be achieved by using distinct cell variations accommodated by vertical or horizontal cell ensembles, suggesting alternative paths for design synthesis and re-synthesis.

The architectural decomposition of the particular cell ensemble (I.A.1.) constituting the mobile phone restraint mat is shown in Figure 10. In this particular example, three cell variations (I.A.1.a., I.A.1.b., and I.A.1.c.) are stacked on top of each other to form an aggregate architectural component, a vertical cell ensemble (I.A.1.). The corresponding functional decomposition illustrates the aggregation of the basic functions that each cell provides, producing aggregate functions such as "aggregate angular expansion", effectively summing up the individual "angular expand" functions of each cell. While some basic functions aggregate in the ensemble, others simply propagate to become functions of the ensemble, such as "connecting to an external air source", "attaching to a flat surface". Also, some functions are absorbed when the ensemble is formed such as "attach to next cell" and "attach to previous cell" which effectively cancel out. For the sake of simplicity, the provisional arrows indicating how basic functions combine to form aggregate functions are no longer color coded according to the subcellular feature category that provided the original basic functions.

\subsection{Unit and Assembly Levels}

The unit level specifies the details of how multiple cell ensembles are combined together to produce a unit, while the assembly level describes how those units can combined to form a more complex assembly. In parallel with the architectural combination, the aggregate functions provided by the ensembles are combined into compound functions at the unit level, and those compound functions combine into technology capabilities provided by the assembly, one or more of which are brought

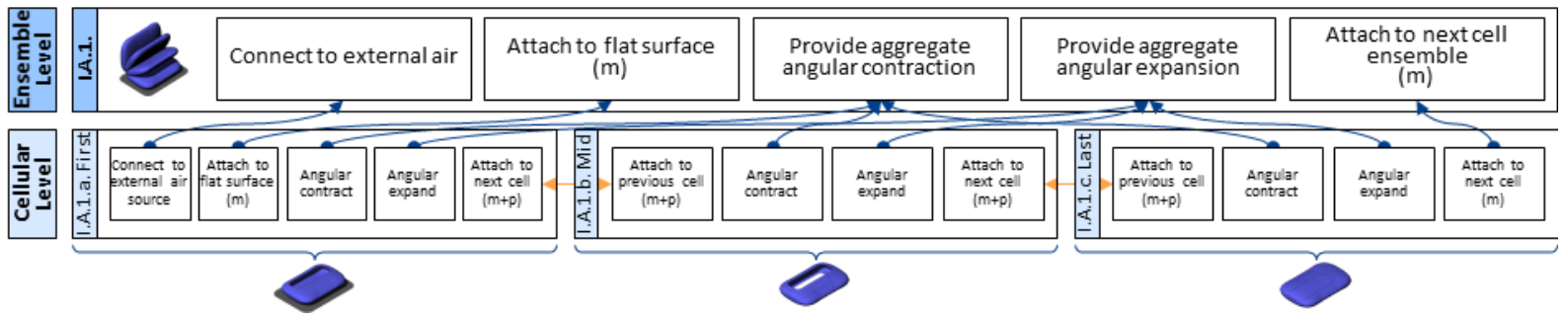

Figure 10. The functional aggregation of cell variations (I.A.1.a., I.A.1.b., and I.A.1.C.) to create a particular ensemble (I.A.1.) providing a set of aggregate functions. 

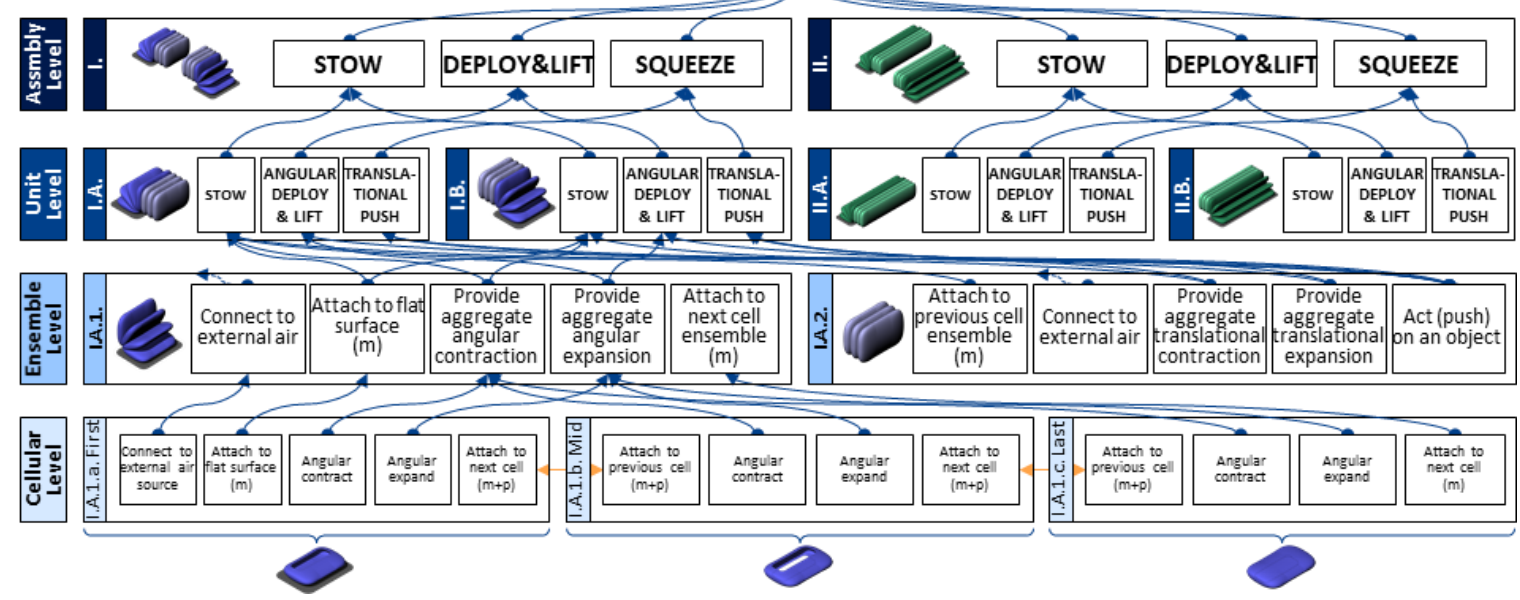

Figure 11. The (partial) functional architectural decomposition of mobile phone restraint mat

together to create the entirety of the constrained layer inflatable system that is being decomposed functionally and architecturally. By assembling multiple units and assemblies together, the compound functions provided by each unit can be distributed in myriad ways and can be activated simultaneously or sequentially to form the technology capabilities.

In the mobile phone restraint mat example (Figure 11), the two cell ensembles shown (I.A.1. and I.A.2.) combine to form one of two identical units (oriented $180^{\circ}$ apart), combining and converting the various aggregate functions into compound functions. For example, "attach to flat surface" and "provide aggregate angular expansion" combine to produce the compound function "angular deploy", while "act (push) on an object" and "provide translational expansion" combine to produce "translational push". Similar to the ensemble level, the "attach to next cell ensemble" and "attach to previous cell ensemble" functions cancel out in the formation of the unit. Functions such as "connect to external air" simply propagate upwards but are not shown for brevity. In this example, the two units I.A. and I.B. combine to form assembly I. which, for example, combines the two "translational push" functions to provide the technology capability "squeeze". Finally, the two assemblies of units I. and II. combine to produce the mobile phone restraint mat system. In Figure 11, the relationship across different levels, including the unit and assembly levels, of the functional architectural decomposition of the mobile phone restraint mat system is shown. For space and readability, only a portion of the cell variations and cell ensembles is exemplified; some branches of the hierarchical tree are left out.

\subsection{Design Implications}

The functional architectural decomposition is a tool geared toward supporting a number of design activities. Conceptual synthesis is strongly supported. For example, by understanding the relationships between sub-cellular structures and the basic functions of a cell, basic functions can be tailored to meet specific needs and new basic functions can be produced. Also, by constructing a library of available cell types, ensemble types, and unit and assembly types with their associated functions, complete systems can be synthesized from existing components. Analysis and redesign are also strongly supported. For example, multiple ways of providing the same higher-level function may exist, and the decomposition can be manipulated by pruning a portion of the hierarchical tree and grafting in an architecturally different, but functionally equivalent replacement to explore design alternatives. Similarly, a design can be simplified by merging or pruning a branch of tree without losing the provided technology capabilities. Thus, the functional architectural decomposition can aid many aspects of the design process, particularly at the conceptual design stage.

\section{CONCLUSION}

This paper introduced constrained layer inflatable systems enabling the systematic design of thin, flat, lightweight, and low-cost inflatable systems that are made of multiple airtight layers, providing various technology capabilities when pneumatically activated. A constrained layer inflatable system consists of 
multiple cell skin layers which create arrangements of interconnected inflatable air cavities, pneumatic ports enabling control over the relative pressure inside the system, and internal or external constraints that restrain the form and create the corresponding functionality of the system. Specific architectural forms are enumerated by exemplifying three constrained layer inflatable systems equipped with distinct technology capabilities, demonstrating a sampling of the wide range of technology capabilities that can be achieved by constrained layer inflatable systems. The embedded reciprocity between the architectural form of a constrained layer inflatable system and the functionality it provides enables the development of functional architectural decomposition approach, which forms the basis for the analysis of the relationship between the hierarchical sets of architectural components and the corresponding hierarchical sets of functions.

The terminology that describes each level of functional architectural decomposition (sub-cellular level, cellular level, ensemble level, and unit and assembly levels) was communicated through decomposing the mobile phone restraint mat system as an example. The interrelated architectural and functional breakdown facilitates the iterative synthesis at the higher levels, where the basic architectural components (i.e. cells) are combined to produce compound ones (ensembles, units, assemblies), matching to the generation of compound functions (aggregate functions, compound functions, and technology capabilities) from the combination of basic ones. This approach empowers designers to generate design alternatives for the design of any constrained layer inflatable system by modifying or substituting its architectural components without affecting the existing functional hierarchy. It also enables the synthesis of new systems given a library of existing functional assemblies, units, ensembles, and cells. While the functional architectural decomposition approach is presented in the context of constrained layer inflatable systems in this paper, it can be further applicable for the analysis, synthesis, and re-synthesis of system designs for many other highly structured architectural classes of systems relying on architectural and functional hierarchies.

\section{REFERENCES}

Follmer, S., Leithinger, D., Olwal, A., Cheng, N., Ishii, H., 2012. Jamming user interfaces: programmable particle stiffness and sensing for malleable and shape-changing devices, in: Proceedings of the 25th Annual ACM Symposium on User Interface Software and Technology - UIST '12. Presented at the the 25th annual ACM symposium, ACM Press, Cambridge, Massachusetts, USA, p. 519. https://doi.org/10.1145/2380116.2380181

Freeland, R.E., Bilyeu, G.D., Veal, G.R., Steiner, M.D. and Carson, D.E., 1997. Large inflatable deployable antenna flight experiment results. Acta Astronautica, 41(4-10), pp.267-277.

Gurt, R., 2015, October. Design and analysis of reinforced rubber membranes for inflatable dams. In Textiles composites and inflatable structures VII: proceedings of the VII International Conference on Textile Composites and Inflatable Structures, Barcelona, Spain. 19-21 October, 2015 (pp. 306-317). CIMNE.

Han, C., Takahashi, R., Yahagi, Y., Naemura, T., 2020. PneuModule: Using Inflatable Pin Arrays for Reconfigurable Physical Controls on Pressure-Sensitive Touch Surfaces, in: Proceedings of the $2020 \mathrm{CHI}$ Conference on Human Factors in Computing Systems. Presented at the CHI '20: CHI Conference on Human Factors in Computing Systems, ACM, Honolulu HI USA, pp. 1-14. https://doi.org/10.1145/3313831.3376838

Matoba, Y., Sato, T., Takahashi, N., Koike, H., 2012. ClaytricSurface: an interactive surface with dynamic softness control capability, in: ACM SIGGRAPH 2012 Emerging Technologies on - SIGGRAPH '12. Presented at the ACM SIGGRAPH 2012 Emerging Technologies, ACM Press, Los Angeles, California, pp. 1-1. https://doi.org/10.1145/2343456.2343462

Mitsuda, T., Matsuo, N., 2005. Shape stabilizer using an articulation-type passive element. Proc. JFPS Int. Symp. Fluid Power 2005, 722-727. https://doi.org/10.5739/isfp.2005.722

Sadeghi, A., Mondini, A., Mazzolai, B., 2019. Preliminary Experimental Study on Variable Stiffness Structures Based on Textile Jamming for Wearable Robotics, in: Carrozza, M.C., Micera, S., Pons, J.L. (Eds.), Wearable Robotics: Challenges and Trends, Biosystems \& Biorobotics. Springer International Publishing, Cham, pp. 49-52. https://doi.org/10.1007/978-3-030-01887-0_10

Seedhouse, E., 2015. Bigelow Expandable Activity Module, in: Seedhouse, E. (Ed.), Bigelow Aerospace: Colonizing Space One Module at a Time. Springer International Publishing, Cham, pp. 87-98. https://doi.org/10.1007/978-3-319-05197-0_5

Sullivan, L.H., 1896. The tall office building artistically considered, first published in Lippincott's 57, March; reprinted in 1947 in Sullivan. Kindergarten Chats and Other Writings.

Terwagne, D., Brojan, M., Reis, P.M., 2014. Smart Morphable Surfaces for Aerodynamic Drag Control. Adv Mater 4.

Tome, B., 2015. Pneumatically Augmenting Inelastic Materials for Texture Changing Interfaces (Doctoral dissertation). Massachusetts Institute of Technology.

Veneman, J., Manterola, I., Bureau, M. and Jung, J.H., 2015. Varstiff, an innovative variable stiffness material, applied in a Wheelchair Positioning Device. In Technically Assisted Rehabilitation Conference, Berlin, Germany (pp. 344-348). 